\title{
ANÁLISIS AXIOLÓGICO DE LA CONSTRUCCIÓN Y VALLDACIÓN DE UN INSTRUMENTO DOCUMENTAL EDUMÉTRICO
}

\author{
AXIOLOGICAL ANALYSIS OF THE CONSTRUCTION AND VALIDATION \\ OF AN EDUMETRIC DOCUMENTARY INSTRUMENT
}

Nelson Paucca Gonzales" ${ }^{*}$, (iD Rusbel Jhon Rafayle Cuadra ${ }^{1}$, (D) Judy Andrea Andrade Torre ${ }^{1}$

nelfilosofia@gmail.com; rjrafayle@gmail.com; judyandradetorre@hotmail.com

${ }^{1}$ Universidad Nacional de Educación, Lima, Perú

*Correspondencia: Nelson Paucca Gonzales. Email: nelfilosofia@gmail.com

Recibido: 26.03.2020 | Aprobado: 09.04.2020

\section{RESUMEN}

La investigación educativa, presenta ciertos sesgos en lo que respecta a la construcción de un instrumento documental de tipo edumétrico y la validación. Esta se debe, a la falta de toma de conciencia axiológica de parte de algunos investigadores, tesistas, asesores y jueces expertos que tienen la obligación de validar el instrumento formulado a través de los ítems. En función al acápite enunciado en líneas anteriores se formula el siguiente objetivo; elucidar la falta de praxis de la ética y moral de las personas involucradas en el proceso de la construcción y validación de un instrumento edumétrico. El análisis del artículo científico corresponde al método analítico - hermenéutico en lo que respecta al enfoque cualitativo y al método estudios instrumentales para el caso del enfoque cuantitativo. El método hermenéutico ha permitido analizar de manera rigurosa el papel que cumplieron las personas mencionadas anteriormente. También, se ha establecido las diferencias de manera categórica entre la construcción de un instrumento de tipo psicométrico y el de tipo edumétrico, esta última se construye a falta de los antecedentes de la investigación a desarrollar y su validez obedece a las teorías formuladas en función a las variables y dimensiones. Finalmente se concluye que las personas involucradas en la construcción edumétrica carecen de la praxis axiológica.

Palabras clave: Axiología, instrumento, validación, edumétrico.

\begin{abstract}
Educational research presents certain biases in what is referred to the construction of an edumetric documentary instrument and its validation process. This may be due to the lack of axiological awareness from some researchers, thesis experts, advisors and expert judges who have the moral obligation to validate the instrument through its items. To summarize what has been exposed previously the following objective is proposed; to notice the lack of ethics and morality of the people involved in the construction and validation process of an edumentric instrument. The analysis of the scientific article corresponds to the analytical - hermeneutical method in what is referred to the qualitative approach and the instrumental studies method in what is referred to the quantitative method. The hermeneutical method has allowed the strict analysis of the role of those who were mentioned before. The results discussion has established the differences between the construction of a psychometric and edumetric instrument, the second one is built due to the lack of previous studies and its validation follows the formulated theories in function to the variables and dimensions. Finally, it is concluded that the people involved in the edumetric construction lack axiological praxis.
\end{abstract}

Keywords: Axiology, instrument, validation, edumetric. 


\section{INTRODUCCIÓN}

El análisis de la construcción y validación de un instrumento documental edumétrico, en tiempos como hoy se ha manifestado a través de una crisis en los diferentes ámbitos de nuestra vida cotidiana y académica. Esta crisis axiológica será abordada desde el plano moral o moralidad, el cual consiste en la ruptura de relaciones interpersonales entre los humanos. En nuestros tiempos un hecho inmoral es visto por los otros como un hecho normal. Estos acontecimientos son problemas de primer orden dentro de nuestra sociedad, naturalmente no puede ser tolerado en el campo académico, puesto que el docente y los investigadores son formados bajo los cánones éticos morales.

El objetivo del presente artículo es elucidar la falta de praxis de la ética y moral de las personas involucradas en el proceso de la construcción y validación de un instrumento edumétrico, a partir de los resultados se propicia la reflexión en los investigadores acerca del rol que cumplen durante el proceso de construcción y validación de un instrumento documental, las mismas que se fundamentan en la praxis de los cánones axiológicos que exige la investigación científica.

En principio el método que ayuda discernir la explicitación del artículo es el de tipo analítico, reflexivo y hermenéutico, el cual inicia con el abordaje de la problemática que se suscita en la construcción de un instrumento documental de medición de tipo edumétrico. En segunda instancia se ha elucidado algunos conceptos relevantes que deben tener en cuenta los investigadores, los jueces validadores y los representantes de la comunidad académica de la investigación. Y en última instancia se ha propuesto las nociones generales y el formato a tener en cuenta al momento de construir un instrumento de medición edumétrico la misma que recoge los aportes del juez validador.

\section{MÉTODOS}

Para el desarrollo del artículo, conviene describir dos métodos fundamentales, puesto que el trabajo es netamente de carácter analítico-reflexivo asociado al enfoque cualitativa. En principio el método que ayuda elucidar la temática es el método hermenéutico, según Ferrater (2001) la hermenéutica se conceptúa como; "La voz Épunveía significa primariamente "expresión (de un pensamiento)"; de ahí explicación y sobre todo interpretación del mismo" (p. 1622). El presente método está estrechamente relacionado con las concepciones de la axiología, durante el desarrollo de la temática se hará notar la intervención de los individuos en el proceso de la validación.

Además de los métodos mencionados, se tiene en el método estudios instrumentales, según Montero y León (2002), el método estudios instrumentales, tiene la finalidad de encaminar el desarrollo de pruebas y apartados, incluyendo el diseño como parte de la adaptación del estudio. Por tanto, este tipo de método está estrechamente relacionado con la construcción de un documento psicométrico, la misma que será ensayado desde la concepción edumétrica que se hace notar en la parte de discusión de resultados, a su vez el método está relacionada con el enfoque cuantitativo.

Se debe tener en cuenta que el método científico, es un procedimiento para tratar un conjunto de problemas. Por tanto, el investigador tiene que apropiarse de ella, para 
desarrollar una investigación a fin de guiar la viabilidad y la obtención de los resultados que busca informar a una determinada comunidad.

\section{DESARROLLO DEL CONTENIDO}

Uno de los problemas que ha podido notar en los últimos tiempos en el campo de la investigación educativa, es el proceso de construcción de un instrumento de medición documental, básicamente se suele notar los problemas que a continuación se detalla:

La falta de responsabilidad acerca de la construcción de un instrumento documental de parte del tesista. Muchas personas quienes realizan tesis no diferencian la función de un instrumento documental. Por lo general, el tesista tiene sus propios objetivos a investigar, a modo de ejemplo se describe la siguiente temática; El diseño curricular y el rendimiento académico en los estudiantes de la institución educativa "Fe y Alegría" de Lima. Respecto al enunciado de la temática, la investigación tiene que abordar entorno a esta problemática, priorizando la elucidación de las variables como es; diseño curricular y rendimiento académico. Sin embargo, el problema surge cuando el tesista pretende desarrollar el capítulo que corresponde al tratamiento de los instrumentos, en este capítulo el tesista orientado por el asesor generalmente suele construir un instrumento. En ese sentido, asesor es el docente responsable de guiar la investigación, Gutiérrez (2019), menciona que el mundo actual requiere de docentes innovadores en el desarrollo de la investigación, queda explicitado que innovar significa mejorar lo ya establecido, generalmente en la praxis cotidiana de los docentes asesores se ha podido nota lo contrario, lo cual es totalmente inaudito por lo siguiente: primero, el hecho de pensar en construir un nuevo instrumento de medición ya es un gran problema y como problema de primer orden necesita ser resuelta, para ello se debe tener en cuenta la estructura de la investigación científica y el método determinado en la investigación. La construcción de un instrumento de medición documental, tiene que ser producto de una investigación exclusivamente de tipo edumétrico. Para la concreción de la temática, es necesario manifestar el estado del arte. La construcción de un instrumento documental de medición edumétrico mínimamente requiere de tres momentos indispensables, con el enunciado no se está afirmando categóricamente que son las únicas, de hecho, que hay otras, lo que se sugiere es tener en cuenta por lo menos aquello. Primero, se formulan los ítems a partir de las variables y las dimensiones las mismas que serán medidas estadísticamente. Segundo, consiste en aplicar el instrumento a los jueces expertos, quienes validarán cada uno de los ítems formulados por el investigador o tesista. Por otra, el instrumento en su conjunto debe ser aplicado al grupo piloto a fin de asegurar la fiabilidad o confiabilidad. Una vez cumplido con las tres fases del proceso, el instrumento quedará a disposición de cualquier investigador que deseen aplicar según su interés. Además, el instrumento aportará a las nuevas investigaciones tal como sostiene Lucas y Miraval (2019), que la investigación tiene la función de describir nuevas teorías o leyes, en el presente caso proponer un nuevo instrumento documental.

Otro de los problemas que se presentan en el proceso de la elaboración de la investigación o tesis, es la carencia de los asesores especialistas en las diferentes temáticas y, a esta se suma la falta de honestidad de quien será responsable de guiar el desarrollo de la investigación o tesis, muchas veces los asesores aceptan los trabajos de los tesistas sin 
conocer a profundidad la temática que se está desarrollado. Por otra parte, existe la imposición de las autoridades en asignar como asesores teniendo en cuenta solo el grado obtenido por el docente, cuando lo indispensables es la especialización en la materia a desarrollar o consolidar. El asesor debe tener en cuenta, que él, es el primer responsable de la investigación (tesis) desarrollado por el graduando.

Respecto a la construcción de los instrumentos documentales de medición edumétria, se debe tener en cuenta que no todos los instrumentos se tienen que construir y en caso de ser así, la tesis debe estar enfocado solo a la construcción de un instrumento de tipo documental de medición edumétrico. Para afrontar esta problemática los docentes que enseñan investigación deben diferenciar las funciones que cumplen un instrumento construido en el ámbito académico.

Desde una concepción general, cabe recalcar que hacer una tesis no necesariamente implica construir un nuevo instrumento, pero tampoco invalida la posibilidad de construir aquello. Lo que debe estar explícito, es que, al hacer una investigación y construir un instrumento el investigador o tesista está asumiendo una doble responsabilidad, el cual no necesariamente tiene que ser así, es decir, no necesariamente el investigador debe cumplir ambas condiciones, puesto que cada uno de ellos son independientes.

Un tercer problema recae sobre los jurados calificadores, en algún momento se ha observado que algunos jurados solo se ha dedicado observar la parte metodológica del trabajo de investigación, cuando su función es quizá analizar de manera crítica los aportes que podría presentar el investigador, frente a este hecho cabe proponer algunas interrogantes ¿Cuáles son los criterios para elegir a un jurado? ¿Quién o quiénes determinan al jurado calificador? Por lo general hemos podido notar que en una sustentación de tesis el que preside aquel acontecimiento es aquel que tiene el grado de doctor y todo gira en esa escala, lo cual parece totalmente obscuro, no necesariamente el quien tenga el grado de doctor pueda garantizar el aporte de la investigación, cualquiera de los jurados calificadores ya sea doctor, magister o licenciado podría avalar el aporte, siempre en cuando que cada uno de ellos se dediquen a la investigación o sean especialistas en la materia presentada por el investigador o tesista.

Por otra parte, aquel que puede garantizar el aporte de una investigación educativa sería aquel individuo que se haya especializado en educación además haya hecho una especialización en investigación científica, estadística, epistemología o filosofía. Si un individuo cumple con una de estas características, se podría decir que su apreciación crítica consolida a la investigación presentada. Con lo sostenido en los acápites anteriores no se está desacreditando la participación y opinión de los individuos que cuentan con el grado de doctor, magister y licenciado. Lo que se está cuestionando es la postura del sistema educativo y a algunas universidades que brindan dichos servicios con una asistencia de cuatro horas a la semana en otros casos con una participación de una vez a la quincena con un horario antipedagógico de 10 horas pedagógicas y a esto se le agrega, que los estudiantes de post grado llevan la asignatura de investigación solo dos veces por ciclo académico.

Un cuarto problema es respecto a la reflexión axiológica en la construcción y validación de un instrumento documental edumétrico, la praxis de la ética moral de parte 
de los jueces expertos son inapropiados, muchas veces los jueces reciben el instrumento a validar y solo colocan la firma y los respectivos datos solicitados cuando realmente su función es hacer una revisión minuciosa a cada uno de los ítems planteados por el tesista, para luego hacer las respectivas sugerencias en caso de ser necesario a fin de consolidar el planteamiento de cada uno de los ítems, para que finalmente se pueda procesar los datos a través del software estadístico.

Finalmente, otro de los problemas es respecto al formato de validación, cada uno de los tesistas utilizan diversos formatos para validar un instrumento documental a pesar de ser de una misma universidad, frente a esta problemática la propuesta es llegar a un consenso para la elaboración de un formato edumétrico entre los diversos especialistas de las diferentes universidades de la facultad de educación, así como también la cantidad de jueces que deben validar el instrumento.

Después de haber presentado el estado del arte, a continuación, se presenta algunos esbozos de tipo conceptual y procedimental en torno al título enunciado, en ese sentido a continuación se elucida lo siguiente:

La axiología, se considera como teoría o filosofía de los valores, además de ser considerado como la disciplina filosófica, esta última es el encargado de analizar los valores como producto de la praxis humana, según Polo (2001) la moral o moralidad es una palabra polisémica ya sea en el uso popular o intelectual, además resalta lo siguiente; "Una primera significación es entenderla como el campo de toda las acciones humanas que pueden ser valoradas como buenas o malas, justas e injustas" (p, 29). La axiología y propiamente dicha la ética me ha permitido hacer una reflexión exhaustiva en torna a la construcción de un instrumento documental de medición de tipo edumétrica, además permite sostener que el problema de la moral ha llegado al punto extremo que se conoce como la inmoralidad en diversas esferas de nuestro entorno. Por tanto, es una necesidad recobrar la importancia de la praxis de la moral y a su vez salvaguardar la crisis de la moralidad por lo menos en el campo de la educación y la investigación.

La crisis de la moral ha generado una ruptura interpersonal entre los hombres de esta sociedad, el valor monetario prima por encima del bien común y lo más agravante es la inconciencia de los profesionales respecto al cumplimiento de sus deberes.

En esta misma línea, se propone algunos aspectos relevantes a tener en cuenta para la construcción y validación de un instrumento. El desarrollo de la construcción y adaptación de un instrumento generalmente debe tener las siguientes características:

Validación del instrumento. - El instrumento debe ser validado por 9 a 11 jueces expertos los cuales deben estar integrados por lingüista, metodólogo, estadístico y por los propios integrantes de la especialidad expertos en la temática. La lógica es, a mayor cantidad de jueces expertos mayor probabilidad y aceptación tendrá el instrumento validado. Por otra parte, el grupo piloto debe estar conformado mínimamente por 30 individuos. Desde la concepción de Arribas (2004), el instrumento a validar la confiabilidad o fiabilidad de un instrumento documental de medición u otro, debe ser aplicado mínimamente entre 30 a 50 personas considerado como el grupo piloto. Por 
ende, se aplica nuevamente la lógica de; a mayor cantidad de grupo piloto mayor será la fiabilidad del instrumento construido.

Nociones generales de la construcción de un instrumento documental de medición edumétrica. - Algunos instrumentos que se pueden construir, se presentan a continuación:

\section{Tabla $\mathbf{N}^{\circ} 1$}

Posibles instrumentos que se pueden construir

\begin{tabular}{|c|c|c|c|}
\hline Instrumentos & Cuestionarios & Escalas & Inventarios \\
\hline Definición & $\begin{array}{l}\text { Es un conjunto de } \\
\text { preguntas y tiene } \\
\text { como objetivo } \\
\text { recoger la } \\
\text { información de lo } \\
\text { que se pretende. }\end{array}$ & $\begin{array}{l}\text { Los ítems propuestos } \\
\text { evalúan si el individuo } \\
\text { está de acuerdo o } \\
\text { desacuerdo con la } \\
\text { información } \\
\text { solicitada. }\end{array}$ & $\begin{array}{l}\text { Es un instrumento } \\
\text { multidimensional, todas las } \\
\text { respuestas son válidas. }\end{array}$ \\
\hline Ejemplo & Un examen & Escala de Liker & $\begin{array}{l}\text { Test de inteligencias } \\
\text { múltiples }\end{array}$ \\
\hline Categoría & $\begin{array}{l}\text { Dicotómica, } \\
\text { presentan solo dos } \\
\text { alternativas } \\
\text { (aprobados o } \\
\text { desaprobados - sí o } \\
\text { no). }\end{array}$ & $\begin{array}{l}\text { Ordinal, presentan } \\
\text { generalmente cinco } \\
\text { alternativas (Nunca, } \\
\text { casi nunca, algunas } \\
\text { veces, casi siempre, } \\
\text { siempre) }\end{array}$ & $\begin{array}{l}\text { Politómica, su función es } \\
\text { medir diferentes capacidades } \\
\text { (Inteligencia: lingüística, } \\
\text { lógico- matemático, visual- } \\
\text { espacial, kinestésica o } \\
\text { corporal-cinética, musical, } \\
\text { interpersonal, intrapersonal y } \\
\text { naturalista) }\end{array}$ \\
\hline
\end{tabular}

(Recuperado y adaptado de https://www.youtube.com/watch?v=9D9PWlFsGhc)

Nota: La tabla $\mathrm{N}^{\circ}$ 1, se evidencia los posibles instrumentos que el investigador puede construir cuando esta no se encuentre en el medio y según la temática de su interés.

Cabe resaltar que un instrumento se construye, cuando esta no existe en el medio, ello implica que la investigación es absolutamente nueva. Por Tanto, carece de antecedentes y de marco teórico. En este sentido, la construcción del instrumento debe partir de un nuevo sustento teórico, no solo se construye el instrumento sino también el marco teórico propuestas por el investigador en función a las variables, dimensiones e indicadores. En el parágrafo anterior se ha mencionado que construir un instrumento implicaba el desarrollo de una tesis y no se puede tomar con ligereza como parte complementaria de una tesis planteado inicialmente. Para no caer en esta paradoja, el investigador o tesista debe partir en principio, a partir de una revisión literaria acerca de la temática que desea desarrollar, a continuación el investigador debe enfocarse en la revisión literaria relacionada con el tema a investigar, para ello se sugiere revisar las diversas tesis existentes tanto nacionales como internacionales en calidad de antecedentes a fin de concordar con uno o varios de los instrumentos utilizados por el autor, está, según las variables determinados por el investigador o tesista. Finalmente, las fuentes literarias y las diversas investigaciones como el que antecede a la investigación deben ser limitada a fin de finalizar con éxito el desarrollo de la investigación, en caso de no encontrar un instrumento que apoye al desarrollo de la investigación, se sugieren optar por realizar una tesis de tipo edumétrico. 
Para el proceso de construcción de un instrumento documental de tipo dimétrico se debe tener en cuenta por lo menos dos momentos:

a. E1 primer momento. - Corresponde al enfoque cualitativo, el cual consiste en plantear cada uno de los enunciados de los ítems de manera coherente y conciso en función a la variable, dimensiones e indicadores.

b. Segundo momento.- Corresponde al enfoque cuantitativo, el cual obedece a la aplicación del proceso estadístico a fin de validar el instrumento documental edumétrico a través del juicio de expertos y el grupo piloto, siempre en cuando el instrumento esté relacionado con el tipo de investigación no experimental y caso de construirse un instrumento para el tipo de investigación expirimental en el campo educativo no es necesario aplicar al grupo piloto, puesto que esta se fundamenta en la propuesta pedagógica, lo cual obedece a la manipulación de las variables a través del grupo experimental. La propuesta pedagógica se plantea a partir de una problemática concreta que estén atravesando los estudiantes a quienes se les denomina como muestra o grupo experimental y su pretensión es mejorar la problemática mediante la aplicación de la propuesta pedagógica durante el desarrollo de las sesiones.

Según Sánchez y Reyes (2015). "La validez es la propiedad que hace referencia a que todo instrumento debe medir lo que se ha propuesto medir, [...]" (p. 167). Desde la concepción de los autores el proceso de validación básicamente pasa por dos momentos tales como:

Validez de contenido, el cual es sostenido por Ñaupas, Valdivia, Palacios y Romero, (2018), de la siguiente manera; "Se refiere al grado como un instrumento refleja un dominio o contenido determinado" (p. 276). Y el segundo es la validez de constructo, el cual se relaciona con el grado de correspondencia entre los resultados de una prueba, en función a las concepciones teóricas en los que se basan los temas que se pretende medir.

Por otra parte, para que el proceso de validación tenga mayor consistencia se sugiere aplicar la estadística $\mathrm{V}$ de Aiken, el cual valida la formulación de cada ítem, siempre en cuando se cumpla la siguiente regla:

- $\quad$ Los ítems se aceptan cuando el $\mathrm{Pv} \geq 0.70$

- Los ítems se rechazan cuando el $\mathrm{Pv}<0.70$

- Dónde: $\mathrm{Pv}=$ es el valor de P. Por tanto, $\mathrm{Pv}=0.70$ como valor mínimo.

Una vez determinado el proceso de validación del instrumento, se pasará a la validación por el grupo piloto, con dicho proceso se determinará el grado de fiabilidad o confiabilidad, para lo cual se sugiere aplicar la estadística Coeficiente Alfa de Cronbach, Celina y Campo (2005), sostienen que en función a la teoría el valor mínimo para aceptar confiable el instrumento es 0.7 ; en caso de ser menor a este valor, la consistencia interna de la escala debe ser rechazada. El instrumento es confiable o fiable siempre en cuando se cumpla los siguientes criterios: 
- $\quad$ El instrumento se acepta cuando el $\mathrm{Pv} \geq 0.70$

- $\quad$ El instrumento se rechaza cuando el $\mathrm{Pv}<0.70$

- Dónde: $\mathrm{Pv}=$ al valor de $\mathrm{P}$. Por tanto, $\mathrm{Pv}=0.70$ como valor mínimo.

(Establecer los modelos explicativos que serán utilizados en la investigación para analizar o intervenir en el problema de investigación)

\section{DISCUSIÓN}

En principio cabe resaltar que el presente artículo, corresponde al enfoque cualitativo por su carácter reflexivo, puesto la pretensión ha sido elucidar el fundamento axiológico en el proceso de la construcción y validación de un instrumento documental edumétrico. Naturalmente el concepto se usa a fin de diferenciar del proceso de construcción de un documento psicométrico, recordemos que la construcción de instrumentos psicométricos está a cargo de los psicólogos, quienes construyen los instrumentos a fin de solucionar problemáticas de su área y dar las posibles soluciones. En función a lo mencionado, cabe responsabilizar que la construcción de un documento de tipo edumétrico responde a las necesidades del campo educativo, según Bisquerra (2018), sostiene que la investigación educativa se organiza y sistematiza con la finalidad de garantizar la calidad del conocimiento obtenido. Por tanto, la construcción del instrumento edumétrico debe garantizar objetivamente los propósitos de la investigación educativa, en ese sentido esta construcción corresponde a los docentes con experticia en la materia. Por otra parte, cabe precisar, que en el campo educativo para obtener conocimientos básicos se hacen bajo los siguientes aspectos; a partir de la investigación no experimental el cual consiste en describir conclusiones generales, también cabe precisar que, para este tipo de investigación se construye el instrumento, y necesariamente deben pasar por la validación por juicio de expertos y confiabilidad o fiabilidad a través del grupo piloto. En la misma línea, queda explicitado que las que las necesidades del docente es diferente a la necesidad del psicólogo. Mientras que los psicólogos trabajan con un determinado grupo de personas de manera personalizada, los docentes trabajan con diferentes grupos de humanos de manera conjunta y su interés está relacionado con el proceso de enseñanza y aprendizaje. Los instrumentos construidos por los docentes están relacionados con el proceso pedagógico, y su finalidad es mejorar la praxis pedagógica relacionada con el uso de las estrategias, métodos y técnicas de enseñanza, aspectos curriculares, procesos evaluativos del estudiante y el desempeño docente, aspectos administrativos relacionadas con los bienes y servicios de las Instituciones Educativas.

Los métodos de la investigación varían de acuerdo a los enfoques, en el presente caso se ha mencionado para ambos casos, en el caso de la investigación cualitativa se ha utilizado el método hermenéutico, por su parte Verd y lozares (2016), sostienen que la investigación cualitativa tiene la función de obtener información a partir de situaciones cotidianos, al cual se le denomina en la investigación científica el abordaje empírico. La investigación cualitativa, permite al investigador describir con mayor propiedad acerca de una propuesta a partir de la experiencia, la misma que finalmente concordará con los sustentos teóricos determinados. Para el caso de la investigación del enfoque cuantitativo, específicamente construcción de un instrumento documental medible, en este caso 
edumétrico se ha usado el método estudios instrumentales, el propósito de la investigación cuantitativa consiste en la medición de las variables, como sostiene Mousalli (2016), que la investigación cuantitativa tiene sus bases en las ciencias naturales y la única forma de llegar a la veracidad de la investigación era a través de la medición de sus variables, en nuestros tiempos estas prácticas se suele notar de manera cotidiana, a tal punto, que los investigadores o tesistas no muestran una diferencia entre desarrollar una investigación y construir un instrumento documental de tipo edumétrico.

Como se ha mencionado en el acápite anterior, los investigadores o tesistas, los docentes de investigación y los asesores no tienen un concepto claro de cuándo o en qué casos se construye un instrumento, queda explicitado que los instrumentos se construyen siempre en cuando que esta no exista en el medio circundante, ello implica la no existencia de antecedentes nacionales o internaciones, por lo que el edúmetra debe construir un nuevo instrumento a partir del sustento teórico de las variables y haciendo uso de la estadística tanto para la validación por jueces y fiabilidad por grupo piloto, siempre en cuando esta sea una investigación del tipo no experimental, en caso de construir un instrumento que midan las variables de tipo experimental el pre y post test debe ser validado por juicio de experto y no necesariamente por el grupo piloto, puesto que el pre y post test no depende de sí mismo, depende de la propuesta pedagógica. El pre test mide la situación diagnóstica de los estudiantes, a partir de los resultados se aplique la propuesta pedagógica con la finalidad de mejorar la situación problemática diagnosticada, esta se verifica a través de la prueba de hipótesis y después de aplicar la prueba post test, este tipo de instrumento valida la propuesta metodológica planteado por el investigador o tesista a través de la mejora del rendimiento académico de los estudiantes u otros.

La praxis de la ética y moral de los jueces expertos y demás miembros mencionados no guarda mayor relación con la teoría que exige los cánones axiológicos de la investigación científica, primero muchos de los asumen esta tarea no tienen la experticia, muchas veces solo emiten las firmas sin haber hecho un análisis de profundidad, quizá el problema se debe a falta de una normativa que clarifique los requisitos con los que deben contar los jueces expertos, asesores entre otros. Empíricamente las instituciones académicas exigen que el juez validador tenga mínimamente el grado de magister y será de mayor relevancia si cuenta con el doctor, estos enunciados son utópicos desde la concepción axiológica, no necesariamente las personas que cuentan con estos grados son idóneas. Además se puede notar de manera empírica y concreta que en las maestrías o doctorados no existe una asignatura de construcción edumétrico, para constatar aquello basta observar la valla curricular de cualquier universidad, lo que es visible en la valla curricular de algunas universidades es el desarrollo de la asignatura de Statistical Package for the Social Sciences (SPSS) traducido al idioma castellano es "paquete estadístico para las ciencias sociales", su objetivo de la asignatura es describir estadísticamente los resultados y constatar la hipótesis a través de la prueba de hipótesis en una tesis. Se tiene en cuenta que las modificaciones en las vallas curriculares se han dado a partir de la exigencias de la Superintendencia Nacional de Educación Superior (SUNEDU) que se creó no hace mayor a cinco años y vienen operando no más de cuatro años, por tanto, una persona con el grado de magister o doctor no necesariamente podría garantizar la validez de un instrumento, es natural el desconocimiento por lo menos en este campo, puesto que no 
se les brindó las herramientas básicas cuando estudiaron. Lo que no estoy de acuerdo es con la actitud de aquellas personas quienes se han hecho dueños de las asignaturas de investigación en general y en las sustentaciones como jurados calificadores se dignen calificar y descafilar, solo en función a la antigüedad de las referencias del marco teórico y antecedentes. Se debe tener en cuenta que la antigüedad de las referencias en lo que respecta al marco teórico es relativo, si bien es cierto, es necesario y es una obligación revisar los teóricos recientes, generalmente lo que se suele encontrar son artículos digitales y los autores de los artículos no necesariamente desarrollan una teoría, sino más bien utilizan algunos conceptos que han propuesto los autores o teóricos clásicos, estos enunciados permite plantear lo siguiente; primero, los artículos pertenecen generalmente al ámbito de los antecedentes en el desarrollo de una investigación lo cual pueden ser considerados de los últimos cinco años o 10 años según crea conveniente una determinada institución. Segundo, los antecedentes del sustento teórico deben estar en relación de lo más reciente a los más antiguo, esta dependerá del investigador mas no del capricho del asesor, una de las reglas en el desarrollo de la investigación científica respecto a la clasificación de las fuentes, la fuente de mayor relevancia es la fuente primaria o de primera mano, es ese sentido, al tener una fuente de tercera o segunda mano, es obligación del investigador o tesista el examinar la fuente primaria o de primera mano. Con ello no sustento de que las otras fuentes no sean válidas para el desarrollo de la investigación. También, es de notarse que la construcción de un nuevo instrumento requiere de nuevas teorías y es posible que estas no se encuentren en la actualidad, lo cual obliga al investigador acudir a los teóricos clásicos a fin de contextualizar y proponer una nueva teoría que respondan a las necesidades del contexto. Otro de los problemas de los jueces expertos, asesores, informantes y jurados calificadores, es el de pretender saber todo, aceptan todo lo que se le presenta y opinan acerca de todo, lo más grave es que las indicaciones de aquellos son irrefutables. Este hecho se califica como la fragmentación de la ética y moral, se sospecha que el ser humano al ser todista va perdiendo el sentido de razonabilidad y la función que cumple la lógica de primer orden, además de negar lo que demanda los cánones axiológicos de la investigación científica.

Los jueces expertos e idóneos para la validación de un instrumento documental de medición deben ser aquellos quiénes han realizado especialización en Estadística e investigación científica, sostengo aquello, puesto que en su valla curricular obligatoriamente existe la asignatura de construcción psicométrico, por tanto, aquellas personas conocen los fundamentos teóricos y prácticos. La formulación de los ítems por el investigador o tesista debe ser revisado por especialistas de la temática, a su vez por los lingüistas, por los metodólogos, estadísticos, epistemólogos, filósofos entre otros. Si el caso fuera así, cada uno de los ítems sería robusto, el cual sería complementado a la aplicación al grupo piloto a fin de constatar la confiabilidad o fiabilidad, finalmente con estos procesos se garantiza la aplicabilidad del instrumento a las diferentes investigaciones relacionadas a la temática.

\section{CONCLUSIONES}

El método hermenéutico permite analizar y elucidar una problemática concreto como es el caso de la axiología y el papel que ejerce este último durante en el desarrollo de la investigación de enfoque cualitativo. 
El método estudios instrumentales permite el desarrollo de los instrumentos básicamente de tipo psicométrico, el cual también nos debe permitir la construcción de un instrumento de tipo edumétrico o adaptación de la misma.

La construcción de un instrumento de tipo documental de tipo psicométrico le corresponde a los psicólogos, econométrico a los economistas y el de tipo edumétrico corresponde a los docentes dedicados a esta materia.

Existe cierta irresponsabilidad de tipo axiológico en el desarrollo de la investigación, básicamente por desconocimiento de funciones, en otros casos de tipo metodológico. Por otra parte, la obtención de los grados no garantiza necesariamente la eficacia de los jurados y asesores.

El trabajo desarrollado por el investigador o tesista necesariamente recae sobre la responsabilidad de los asesores, puesto que su función es acompañar y guiar el desarrollo de la investigación.

La expresión crisis moral, refiere a la ruptura de las relaciones interpersonales entre los hombres, la misma que debe ser analizada y fortalecida durante el desarrollo de la investigación a través de los resultados como producto final.

Para el campo de la investigación educativa, necesariamente el instrumento que se construye debe ser de tipo edumétrico, en la mayoría de las investigaciones se ha podido notar que las tesis suelen utilizar un instrumento de tipo psicométrico. La aplicación de un instrumento psicométrico de parte de los docentes a los estudiantes de nivel básica o superior no aporta al campo de la educación. La aplicación de instrumento psicométrico es posible, sin embargo, necesariamente se deben adaptar al campo educativo.

Solo se construye un instrumento documental de medición de tipo edumétrico, siempre en cuando esta no exista en el medio circundante. Esta construcción edumétrica debe ser desarrollada y validada por los expertos en la materia.

La construcción del instrumento documental de tipo edumétrico debe nacer de sus propias necesidades, mas no debe ser un complemento de una tesis formulada inicialmente de tipo experimental o no experimental.

La estadística juega un papel importante en la construcción documental de tipo edumétrico, tanto en el proceso de validación y el proceso de fiabilidad o confiablidad.

Es una necesidad la existencia de una estructura o formato para la construcción y validación de un instrumento documental de tipo edumétrico, la misma que debe nacer a partir del consenso de los representantes de las diferentes universidades.

La construcción de un instrumento documental de tipo edumétrico debe pasar obligatoriamente primero por los jueces expertos y posteriormente por el grupo control a fin avalar la consistencia y aceptabilidad del instrumento.

Los jueces avalan la validez cada uno de los ítems formulados por el investigador o tesista, mientras que el grupo piloto avala la confiablidad del instrumento en su conjunto. 


\section{REFERENCIAS BIBLIOGRÁFICAS}

Arribas, M. (2004). Diseño y validación de cuestionarios. Recuperado de: http://www.enferpro.com/documentos/validacion_cuestionarios.pdf.

Bisquerra, R. (2019). Metodología de la investigación educativa (6 edición). Madrid: Muralla.

Bojórquez, J., López, L. y Hernández, M. (2013). Utilización del alfa de Cronbach para validar la confiabilidad de un instrumento de medición de satisfacción del estudiante en el uso del software Minitab. Recuperado de: http://laccei.org/LACCEI2013-Cancun/RefereedPapers /RP065.pdf.

Celina, H. y Campo, A. (2005). Aproximación al uso del coeficiente alfa de Cronbach. Recuperado de http://www.scielo.org.co/pdf/rcp/v34n4/v34n4a09.pdf.

Ferrater, J. (2001). Diccionario de filosofía. (Tomos I, II, III y IV). Barcelona: Arial.

Gamarra, G., Berrospi, J., Pajuelo, O y Cuevas, R. (2008). Estadística e investigación. Lima: San Marcos.

Gutiérrez, V. (2019). Técnica de exposición-discusión y formación cientifica en estudiantes de pregrado. Recuperado de http://revistas.unheval.edu.pe/index.php/riv/article/view/ $345 / 323$

Hernández, R. (2014). Metodología de la investigación (6ª edición). México: Mc. Graw Hill.

Lucas, A y Miraval, C. (2019). Perspectiva epistemológica de las matemáticas como fundamento de las ciencias. Recuperado de http://revistas.unheval.edu.pe/index.php/riv/article/ view/170/175

Martínez, C. (2012). Estadística y muestreo. (10ª edición). Bogotá: Ecoe.

Mias, C. (2018). Metodología de investigación estadística aplicada e instrumentos en neuropsicología. Argentina: Encuentro.

Montero, I. y León, O. (2002). Clasificación y descripción de las metodologías de investigación en Psicología. Recuperado de: http://www.aepc.es/ijchp/articulos_pdf/ijchp-53.pdf.

Mousalli, G. (2016). Métodos y diseños de investigación cuantitativa. Recuperado de https://www.researchgate.net/publication/303895876_Metodos_y_Disenos_de_ Investigacion_Cuantitativa.

Ñaupas, H., Valdivia, M., Palacios, J. y Romero, H. (2018). Metodología de la investigación cuantitativa - cualitativa y redacción de tesis. ( $5^{\text {ta }}$ edición). Bogotá: $\mathrm{U}$.

Polo, M. (2001). Ética modo de vida, comunidad y ecología. Lima: Mantaro.

Sánchez, H. y Reyes, C. (2015). Metodología y diseños en la investigación cientifica. (5a edición). Lima: Business Support Aneth.

Verd, J. y Lozares, C. (2016). Introducción a la investigación cualitativa fases, métodos y téenicas. España: Sintesis. 